\title{
HIGHLIGHTS
}

RHEUMATOID ARTHRITIS

\section{Monitoring serum concentration of infliximab might improve RA disease control}

Response to therapy with the tumor necrosis factor inhibitor infliximab can vary among patients with rheumatoid arthritis, owing at least in part to differences in individual pharmacokinetics. Mulleman and colleagues demonstrate that taking into account infliximab concentration when making dose-adjustment decisions could lead to better control of clinical disease activity in these patients.

The observational, open-label study involved 24 patients at a single center in France who had been receiving a stable dose of infliximab before the study start. Participants were followed over four consecutive visits at which they received an infusion of infliximab. As well as clinical assessment, investigations at each visit included testing for anti-infliximab antibodies and serum concentration of infliximab by use of enzyme-linked immunosorbent assay.

At the first visit, one of four therapeutic strategies was selected on the basis of 28-joint Disease Activity Score and physician's global assessment of disease activity: decrease the infliximab dose, maintain the dose and possibly add another therapy, increase the infliximab dose, or discontinue infliximab and switch to a different therapy. At visit 2, one of these four options was again selected, but with consideration of the trough serum infliximab concentration measured at visit 1 as well as disease activity control.

\section{4 ... measurements of serum} concentrations of the drug could be used to modify individual dose-adjustment decisions... 77

In total, the therapeutic decision was changed on the basis of infliximab concentration for 12 patients (50\%). Seven patients received a dosage increase at the second visit, six of whom had inadequately controlled disease and medium or low serum infliximab concentration. By contrast, this strategy was selected for only three patients at the first visit.

DAS28 scores for patients who received a dosage increase improved considerably between visits 1 and 3; this improvement was maintained at visit 4 .

Notably, changes in DAS28 score and infliximab concentration between visits 1 and 4 were inversely correlated.

Although the results of this small study of patients undergoing stable, longterm infliximab therapy for rheumatoid arthritis should be interpreted with caution, they suggest that measurements of serum concentrations of the drug could be used to modify individual doseadjustment decisions, and might improve control of disease activity.

Sarah Price

Original article Mulleman, D. et al. Infliximab concentration monitoring improves the control of disease activity in rheumatoid arthritis. Arthritis Res. Ther. 11, R178 (2009) 